\title{
A METHOD OF GENERATING DEM FROM DSM BASED ON AIRBORNE INSAR DATA
}

\author{
Wenqing $\mathrm{Lu}^{1,2, *}$, Jixian Zhang ${ }^{1,2}$, Guochao Xue ${ }^{1,2}$, Chenggang Wang ${ }^{1,2}$ \\ ${ }^{1}$ Shan dong University of Science and Technology, Qingdao 266590, China-(luwenqing2018, xgc7177)@163.com, \\ zhangjx@casm.ac.cn,wcg2049@gmail.com \\ ${ }^{2}$ Key Laboratory of Geo-Informatics of State Bureau of Surveying and Mapping, Chinese Academy of Surveying and Mapping, \\ Beijing100830, China
}

KEY WORDS: InSAR, DSM, Adaptive Threshold Segmentation, DEM, Filtering

\begin{abstract}
:
Traditional methods of terrestrial survey to acquire DEM cannot meet the requirement of acquiring large quantities of data in real time, but the DSM can be quickly obtained by using the dual antenna synthetic aperture radar interferometry and the DEM generated by the DSM is more fast and accurate. Therefore, it is most important to acquire DEM from DSM based on airborne InSAR data. This paper aims to the method that generate DEM from DSM accurately. Two steps in this paper are applied to acquire accurate DEM. First of all, when the DSM is generated by interferometry, unavoidable factors such as overlay and shadow will produce gross errors to affect the data accuracy, so the adaptive threshold segmentation method is adopted to remove the gross errors and the threshold is selected according to the coherence of the interferometry. Secondly DEM will be generated by the progressive triangulated irregular network densification filtering algorithm. Finally, experimental results are compared with the existing high-precision DEM results. The results show that this method can effectively filter out buildings, vegetation and other objects to obtain the high-precision DEM.
\end{abstract}

\section{INSTRUCTION}

Interferometric synthetic radar, InSAR technology plays an important role in earth observation. It can perform large-scale and high-precision measurement on the ground all-weather and allday. Therefore, InSAR technology has become one of the means to acquire DEM quickly and accurately. Airborne dual-antenna radar can avoid the problem of phase-in-phase decoherence in repeated orbital interference, so it has a great advantage in obtaining terrain data. In this study, a series of surface elevation models including surface buildings, bridges, and trees are obtained through a series of interference processes based on the airborne dual-antenna SAR data. In terms of unavoidable gross errors such as shadows and overlaps, an adaptive threshold segmentation method is proposed to effectively remove the gross errors, and the removal of gross errors has a higher accuracy than traditional measurement. The generation of DEM uses an irregular triangulation network progressive encryption filtering method. The filtering process is carried out through TIN triangle iterative encryption. When no new point is added to the triangulation network, the operation ends. This method removes the feature points and only retains the ground points.

\section{THE PRINCIPLE OF OBTAINING DSM THROUGH INSAR}

Cross-track interferometry mode of operation is a fixed antenna mounted on an aircraft perpendicular to the direction of flight. It can accurately obtain surface dimensional information. The following describes the principle of interferometric SAR measurement in the Cross-track interferometry mode of operation. Figure 1 shows the geometric relationship of interference SAR.

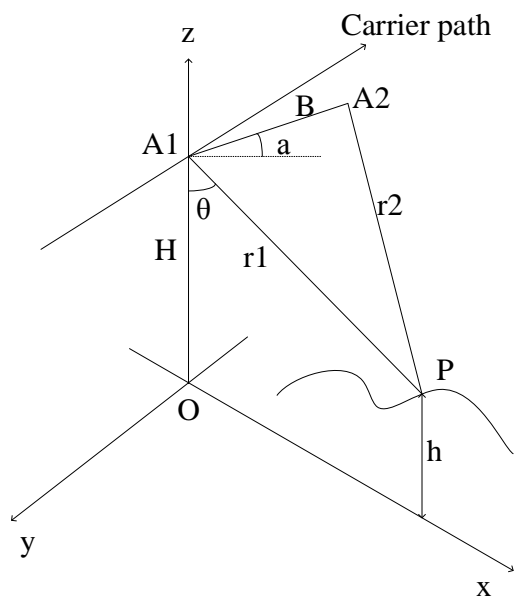

Figure 1. Figure placement and numbering

$\mathrm{A}_{1}$ and $A_{2}$ are primary and secondary antennas on the flight platform, and $\mathrm{B}$ is baseline vector, $\alpha$ is baseline angle, and $\theta$ is the angle of observation at the ground target point $\mathrm{P}$.

The phase difference calculated by radar complex image pair is:

$$
\varphi=\frac{2 \pi Q}{\lambda}\left(r_{2}-r_{1}\right)
$$

In the formula above, $\lambda$ is radar wavelength; $Q$ is working mode of the antenna; when working in "standard mode", $\mathrm{Q}=1$, When working in "ping-pong mode", $\mathrm{Q}=2 ; r_{1}$ and $r_{2}$ correspond to the slant distance of the antenna at $\mathrm{A}_{1}$ to point $\mathrm{P}$ and $A_{2}$ to point $\mathrm{P}$.

\footnotetext{
* Corresponding author at: College of Geomatics, Shandong University of Science and Technology, Qingdao.

E-mail address: luwenqing2018@163.com.
} 
The target value $\mathrm{h}$ of the ground target point $\mathrm{P}$ is:

$$
\mathrm{h}=\mathrm{H}-r_{1} \cos \theta
$$

In the triangle $\mathrm{A}_{1} A_{2} P$, calculated by the cosine theorem:

$$
\sin (\theta-\alpha)=\frac{r_{1}^{2}+b^{2}-r_{2}^{2}}{2 r_{1} b}
$$

From the above three basic relational expressions, the elevation information $h$ of the target point for interferometric SAR measurement is given as:

$\mathrm{h}=\mathrm{H}-r_{1}\left\{a-\arcsin \left[\frac{\left(\frac{\lambda \varphi}{2 \pi Q}+r_{1}\right)^{2}-b^{2}-r_{1}^{2}}{2 r_{1} b}\right]\right\}$

\section{THE GENERATION OF DSM}

The following figure 2 shows the process flow of DEM data generation using airborne InSAR data:

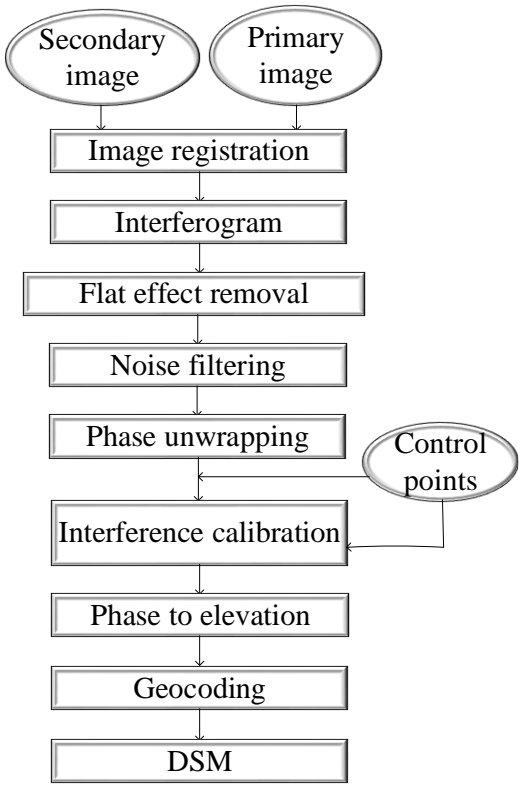

Figure 2. The flow chart of generation DSM using airborne InSAR data

\subsection{Image registration}

Because there is a certain rotation, translation and scaling between the two acquired SAR images, the pixels in the same location do not correspond to the same ground scattering unit. Therefore, the main and auxiliary images need to be accurately registered and resampled before obtaining the interferometric phase.

Image registration is usually divided into two processes: pixel registration and sub-pixel registration. Pixel registration is a coarse registration. Two images of the entire scene or two images of a large area are registered using the same translation amount. This translation can be calculated using radar and observation geometry. The purpose of coarse registration is to reduce the subpixel registration search area and greatly reduce the amount of sub-pixel registration operations. Sub-pixel registration is to evenly divide the image after the rough registration into several small blocks, and finely register each small block one by one.

\subsection{Interferogram}

The conjugate multiplication of the primary and secondary images corresponding to the pixel values can result in the phase difference at each pixel to form an interferogram.

Complex image 1:

$$
u_{1}=\left|u_{1}\right| e^{j \varphi_{1}}
$$

Complex image 2:

$$
u_{2}=\left|u_{2}\right| e^{j \varphi_{2}}
$$

Interferogram is:

$$
u_{\text {int }}=u_{1} u_{2}^{*}=\left|u_{1}\right|\left|u_{2}\right| e^{j\left(\varphi_{1}-\varphi_{2}\right)}
$$

Coherence is used to measure the degree of similarity between images and the quality of interference fringes.

$$
\gamma=\frac{\left|E\left[u_{1} u_{2}^{*}\right]\right|}{\sqrt{E\left[\left|u_{1}\right|^{2}\right] E\left[\left|u_{2}\right|^{2}\right]}}
$$

$\gamma$ is between the 0 and 1 , the closer to 1 , the higher the coherence.

\subsection{Flat effect removal}

The phase difference at any two points in the interference fringe diagram consists of two parts: one is the phase difference between two points on the flat ground; and the other is phase difference caused by the height difference between the two points Therefore, the flat phase difference is removed to obtain a high differential phase difference.

The specific elimination method generally uses a reference earth surface and an interference baseline model to simulate the ground phase, and then subtract the equivalent from the original interference map to achieve the purpose of removal. The flat effect elimination mainly uses the flattening method based on orbital parameters

\subsection{Noise filtering}

Due to the system noise generated during the SAR imaging process, the filtering method is used to both filter out noise interference and maintain the continuity of interference fringes. The specific algorithm is as follows:

1.Interferogram $\mathrm{I}(x, y)$ is FFT transformed to frequency domain FFT $\mathrm{I}(u, v)$, so local power spectrum energy $\mathrm{S}(u, v)$ can be obtained.

2.Power spectrum energy convolution with a $3 \times 3$ smooth window is:

$$
\hat{S}(u, v)=S(u, v) \otimes \mathrm{W}(u, v)
$$


3.Exponentially computing the power spectrum:

$$
\mathrm{Z}(u, v)=[\hat{S}(u, v)]^{\alpha}
$$

4.Perform inverse FFT operation to obtain filtered interference phase:

$$
\hat{I}(x, y)=F F T^{-1}\{\hat{I}(u, v) Z(u, v)\}
$$

\subsection{Phase unwrapping}

The phase value in the interferogram is the main value of the interferometric phase. And value range is $[-\pi, \pi)($ or $[0,2 \pi))$. A step jump will occur at the interference fringe edge at the interferometric phase value. The phase value changes from $-\pi$ to $\pi$, or from $\pi$ to $-\pi$, the phase of the interference before and after the jump differs by an integer period. In order to calculate the elevation value of the ground point from the interference phase, the entire interferogram must be determined. The relative number of phases between the interferometric phases is the phase unwrapping of the interferogram. The interference phase generated by the interference image is a winding phase with a period of $2 \pi$ and the phase unwrapping is to restore the winding phase to the real phase.

\subsection{Interference calibration}

The purpose of interfering SAR calibration is to correct the phase shift and interference parameter deviation of the system equipment to improve the accuracy of generating DEM. The correction of phase shift of the system equipment is called equipment calibration of the interference system, and the interference parameters of the deviation is called the interference parameter calibration. Interferometric parameter calibration includes two methods: interference calibration based on sensitivity equation and interference calibration based on phase correction.

\subsection{Geocoding}

The elevation calculated by InSAR only obtains a set of ground elevation values corresponding to each point of the reference image. The elevation value is based on the SAR image coordinate system, and the SAR image coordinates must be converted into a more general reference coordinate system can be used for practical applications of DEM and SAR images.

Geocoding has mainly been positioned and resampled in two steps. The positioning is divided into two kinds: direct positioning and indirect positioning. These two kinds of positioning are all adopted RD model, and direct positioning is divided into numerical solution method and analytical algorithm according to different solving methods. The representative numerical solution is ASF, and the analytic algorithms are RGM and AGM.

\section{THE ADAPTIVE THRESHOLD SEGMENTATION METHOD}

The DSM gross error processing is to remove the dark noise in the original DSM generated by the InSAR and an outlier that is significantly lower than the ground. The coherence of interferometric measurements is influenced by factors such as the time interval of interferometric pairs, spatial separation, data processing, and system noise. According to the causes, decoherence can be divided into baseline decoherence, temporal decoherence, bulk scattering decoherence, noise decoherence, and data processing decoherence. The longer the interval, the lower the degree of coherence; the shorter the space baseline, the higher the degree of coherence. The difference in coherence associated with the time interval is mainly due to changes in the scattering characteristics of the underlying surface. The two antennas of the airborne dual-antenna InSAR system are simultaneously observed, so the time-correlation problem of the heavy rail interference mode can be avoided. And because its space baseline is very short, if the machine ignores the decoherence of the noise, the dual-antenna InSAR complex image has very high coherence. However, in shadowed, overlaid areas with little echoes, no echoes, or areas where strong targets are scattered, InSAR images exhibit low coherence. Therefore, selecting thresholds based on image coherence can eliminate some gross errors. Therefore, the main steps of the adaptive threshold segmentation algorithm are as follows:

1. Select a window of a certain size in the coherence coefficient graph, count the value of the coherence coefficient in this window, and calculate the statistics such as the mean, median, and standard deviation of the coherence coefficient values.

2.Automatically determine the size of the segmentation threshold based on the statistics of the coherence coefficient in the statistics window. The threshold is the mean minus a few times the standard deviation of the coherence coefficient:

$$
\gamma^{T}=\bar{\gamma}-n * \sigma, \mathrm{n} \text { is } 0,1,2,3 \ldots
$$

According to the threshold obtained by the above steps, the DSM data is thresholded to remove gross errors. The processing function is:

$$
h^{\prime}=\left\{\begin{array}{l}
h, \gamma>\gamma^{T} \\
0, \gamma<\gamma^{T}
\end{array}\right.
$$

The point where the coherence coefficient is larger than the threshold value and the height is not changed; the point where the coherence coefficient is smaller than the threshold value, and the elevation value is null.

\section{THE PROGRESSIVE TRIANGULATED IRREGULAR NETWORK DENSIFICATION FILTERING ALGORITHM}

\subsection{Fundamental}

The irregular triangulation network organizes the point cloud data through a certain mathematical method, and divides the entire point cloud area into triangle areas that do not overlap each other. The surface of the terrain is fitted with triangles connected to each other. The optimized triangular mesh can automatically adjust the density of the triangle according to the change of the curvature of the point. In regions where the topography changes greatly, the more densely constructed triangle mesh is more delicate. The triangular network with a small change trend and flat terrain is rough. In this way, it is one of the main methods for the application of point cloud data that both avoids the surplus of data and guarantees better preservation of ground information.

The idea of progressively encrypting TIN is to construct a sparse triangulation network through seed kernel points, and iteratively encrypts TIN layer by layer. Seed TIN is initially below the encryption network, the slope of TIN's surface is affected by the network configuration parameters, and progressively encrypted 
TIN continues to fit real discrete discontinuous points under parameter constraints.

Assuming that the local area of the terrain is flat, we select the lowest point in the local area as the seed point to construct the initial triangulation, and determine whether the point is a ground point according to the height included angle and vertical distance from any point in the triangle network to the nearest vertex of the triangle. If it is a ground point, TIN encryption is performed until there does not exit new ground points. The algorithm is suitable for complex urban areas and can handle terrain discontinuities. The progressive encryption filtering method distinguishes the ground point from the non-ground point by performing a process of "encryption of the foot point on the ground" and "point cloud foot point" through multiple iterations. The maximum number of encryptions ends when the encryption process is performed until there is no new ground foot point or the number of encryptions reaches a preset level.

\subsection{The Algorithmic Foundation of the Progressive} triangular filter algorithm

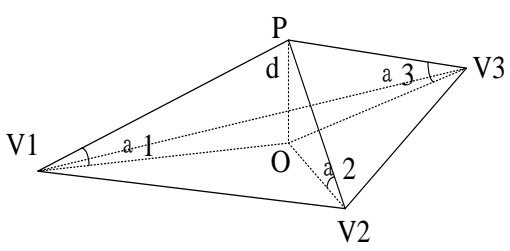

Figure 3. Progressive triangular filter geometry diagram

As shown in the figure 3, $\mathrm{P}$ is a discrete point, $V_{1} 、 V_{2}$ and $V_{3}$ are the three vertices of the triangle, $\mathrm{d}$ is the distance from point $\mathrm{P}$ to the triangle plane, and the foot is $\mathrm{O}$, and $\alpha_{1} 、 \alpha_{2}$ and $\alpha_{3}$ are the angle formed by the point $\mathrm{P}$, the vertices of the triangle, and the feet. $\alpha_{1} 、 \alpha_{2}$ and $\alpha_{3}$ are defined as iteration angles, and $\mathrm{d}$ is defined as repetition distance. The progressive triangulation filtering algorithm uses the repetition angle and the repetition distance as the filtering parameters, and continuously extracts the new ground point by encrypting the triangulation network.

The three plane vertices $V_{1} 、 V_{2} 、 V_{3}$ of the triangle determine the plane equation as:

$$
A x+B y+C z+D=0
$$

The distance $\mathrm{S}$ between the triangle vertices $V_{1}, V_{2}, V_{3}$ and the point $\mathrm{P}$ is

$$
\begin{gathered}
S_{i}=\sqrt{\left(X_{i}-X_{P}\right)^{2}+\left(Y_{i}-Y_{P}\right)^{2}+\left(Z_{i}-Z_{P}\right)^{2}} \\
i=1,2,3
\end{gathered}
$$

The repeated distance $\mathrm{d}$ and the repetition angle $\alpha$ are:

$$
\begin{aligned}
& d=\left|\frac{A x_{p}+B y_{p}+C z_{p}+D}{\sqrt{A^{2}+B^{2}+C^{2}}}\right| \\
& \alpha_{i}=\arcsin \frac{d}{s_{i}}, \quad i=1,2,3
\end{aligned}
$$

Repeated angles and repeated distances reflect the degree of triangulation uplift. The smaller the iteration angle is, the lower the probability of the low-vegetation point on the ground being misclassified as the ground point; the repeated distance ensures that when the triangle is large, the triangulation does not cause large jump upwards, and it can effectively filter some low-rise buildings. The basic idea of this algorithm is:

First, select the seed point, and divide the points in the area into regular grids with large unit spacing. The size of the grid is based on the largest structure type of the area, for example, $60 \times 60$ meters is a unit;

Then find the lowest point in each grid as the initial seed point, and use these initial seed points to generate a sparse TIN; Afterwards, the TIN encryption will continue to be added to meet the conditions;

Finally, the TIN is located below these points, and the curvature of the TIN is limited by parameters. In the encryption process, if the vertical distance $\mathrm{d}$ from a point to the nearest triangular surface and the maximum angles $\alpha_{1} 、 \alpha_{2}$ and $\alpha_{3}$ between the point and the nearest vertex, the triangle are less than the set threshold, the point is added to the ground point. The collection then recalculates the TIN and then discriminates the points in the non-ground point set. This iterative process deals with layer-bylayer encryption until all points are traversed and no new ground points are added. The original point cloud data is divided into two classes, ground points and non-ground points.

\section{TEST SITES AND DATA SETS}

The orthophoto of the test area is shown in Figure 4. This article selects airborne InSAR data from the Zoige area in Sichuan Province. It ranges from 2900 to $4200 \mathrm{~m}$ and the height difference above $1000 \mathrm{~m}$. It belongs to the mountain terrain. The direction of flight is from east to west and from west to east. There are many kinds of features in the area, such as vegetation, roads, houses and so on. This data was selected by the Chinese Academy of Surveying and Mapping CASMSARV2 system the $\mathrm{X}$-band airborne dual-antenna SAR. The SAR system platform parameters for data acquisition are shown in Table 1.

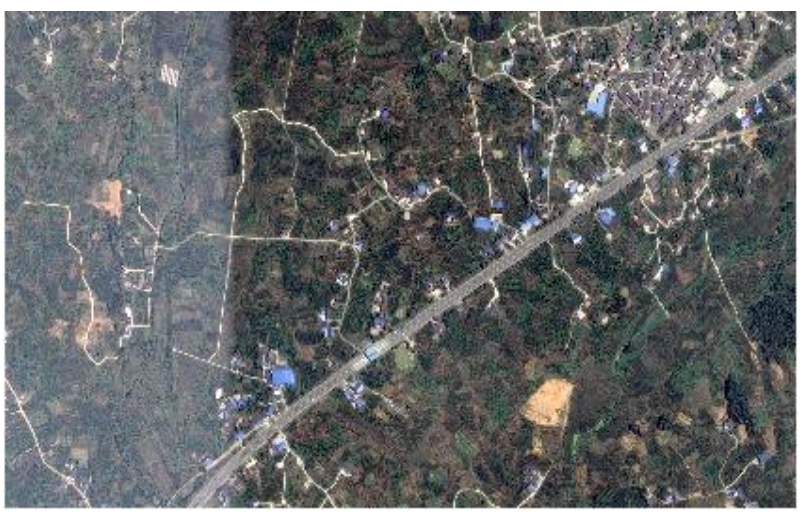

Figure 4. Orthophoto map of the test area

\begin{tabular}{ll}
\hline Parameter & Instruction \\
\hline Baseline length/m & 2.20 \\
\hline Radar operating frequency/GHz & 9.6 \\
\hline Platform relative flying height $/ \mathrm{m}$ & 2686 \\
\hline Resolution $/ \mathrm{m}$ & 0.5 \\
\hline Initial slant range/m & 2344.86 \\
\hline Operating mode & $\begin{array}{l}\text { Single transmitter and } \\
\text { dual receiver }\end{array}$ \\
\hline Average ground elevation $/ \mathrm{m}$ & 2800 \\
\hline \multicolumn{2}{c}{ Table 1. The SAR system platform parameters }
\end{tabular}


A coherence coefficient map is obtained after SAR interference processing, as shown in Figure 5.

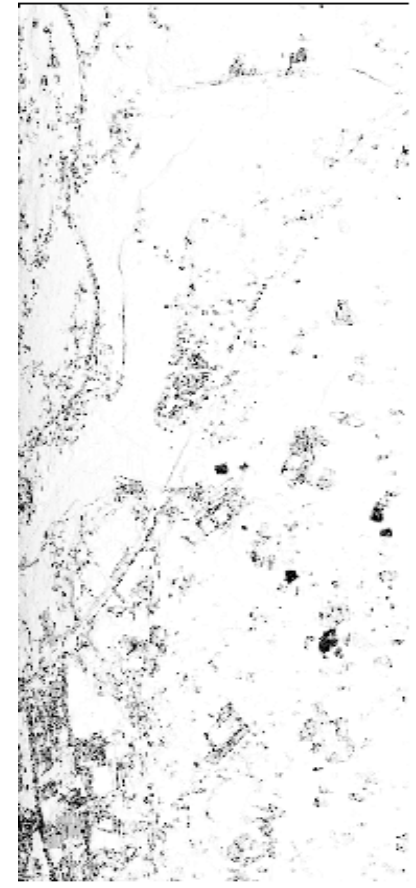

Figure 5. Coherence coefficient map

The adaptive threshold segmentation is performed on the coherence coefficient graph to remove the effect of gross errors, so that the threshold $n=1$ and the window size is $3 \times 3$. The elevation map acquired after adaptive threshold segmentation is shown in Figure 6. It can be seen from the figure that the adaptive threshold segmentation method can filter out some gross errors.

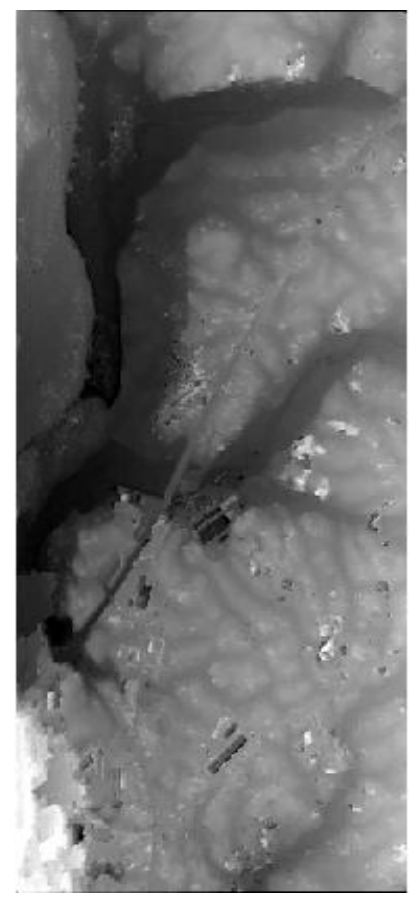

(a)

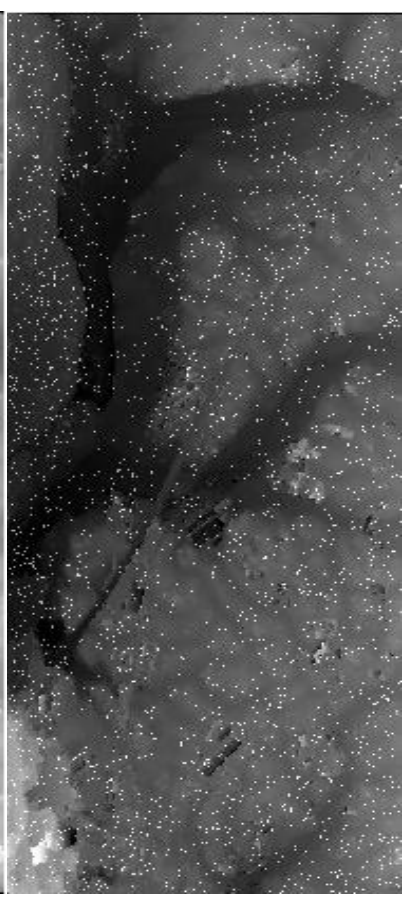

(b)
Figure 6. (a) Elevation map without adaptive threshold segmentation; (b) Elevation map with adaptive threshold segmentation
Afterwards, the DSM in this area was obtained by geocoding, as shown in Figure 7.

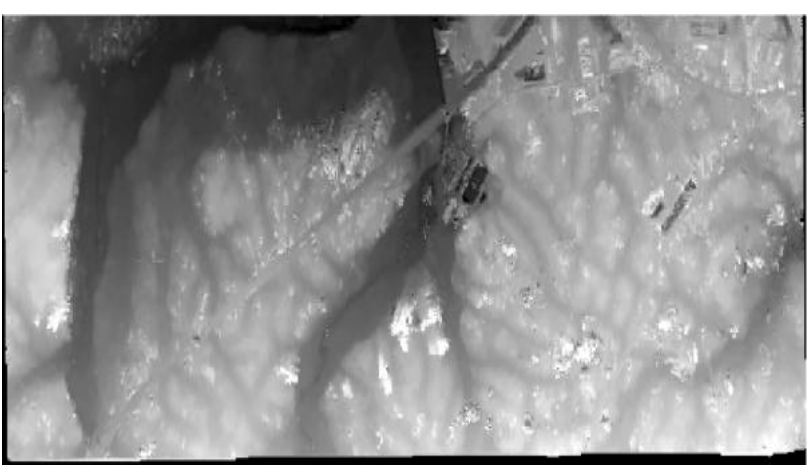

Figure 7. DSM

The DSM extraction point cloud data is progressively encrypted and filtered by an irregular triangulation network. First, a sparse TIN is generated, and then the point satisfying the condition is continuously added to the TIN encryption. The TIN is shown in Figure 8. Filtering generates DEM as shown in Figure 9.

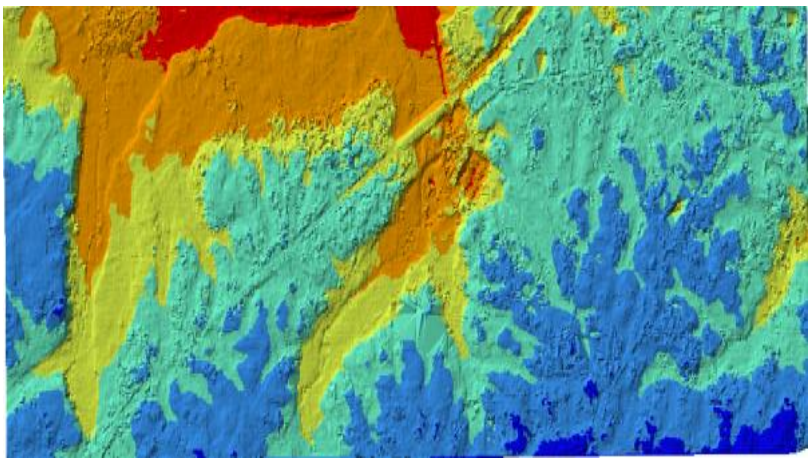

Elevation (m)

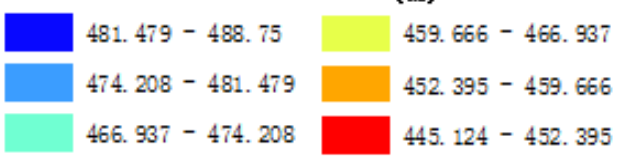

Figure 8. TIN

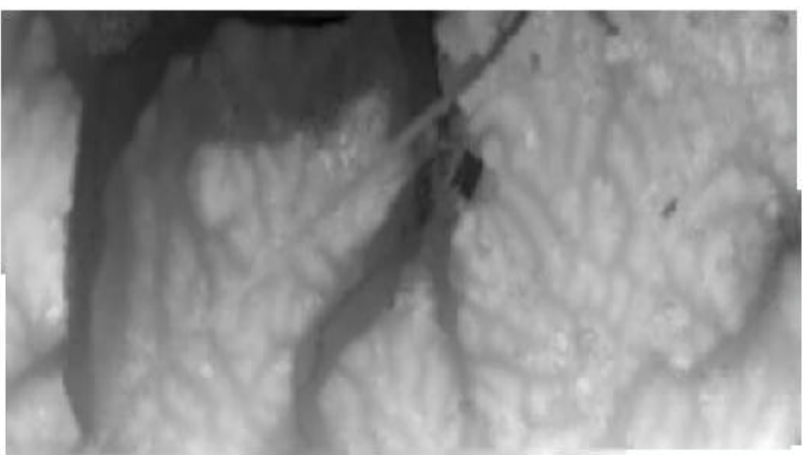

Figure 9. DEM

\section{RESULT AND ANALYSIS}

The purpose of the accuracy assessment is to check the accuracy of DEM. The conventional checkpoint method is a point on the checkpoint map. This one-to-one correspondence method is suitable for the evaluation of digital mapping. This paper 
examines the elevation accuracy of the DEM and therefore requires a checkpoint to correspond to the DEM local position for evaluation. Select 4 GPS control points as checkpoints on the existing DSM, and the height is $\mathrm{H}$ and find the position of the newly generated DEM corresponding point, select points near this, and the elevation value is $H_{i}$, and compare them one by one with the checkpoint to calculate the squared difference of elevation, denoted by RMSE. The DEM accuracy can be assessed very well.

$$
R M S E=\sqrt{\frac{\sum_{i=1}^{n}\left(H-H_{i}\right)^{2}}{n}}
$$

\begin{tabular}{ccccc}
\hline Point number & X coordinate & Y coordinate & Actual elevation value & RMSE \\
\hline $\mathbf{5 6 0 1}$ & 34643874.659 & 3321511.104 & 466 & 0.98 \\
\hline $\mathbf{5 6 0 2}$ & 34643539.386 & 3321295.571 & 472 & 1.24 \\
\hline $\mathbf{5 6 0 3}$ & 34643707.023 & 3321311.537 & 465 & 0.85 \\
\hline $\mathbf{5 6 0 4}$ & 34644018.348 & 3321319.519 & 470 & 0.96 \\
\hline
\end{tabular}

Table 2. DEM accuracy evaluation

Experimental results show that the adaptive threshold segmentation method can filter out some obvious gross errors and guarantee the accuracy of the subsequent generated DEM. Irregular progressive encryption filtering algorithm not only can overcome the limitation of the traditional algorithm only for certain specific continuous surface areas, but also has applicability for urban terrain and point cloud data in complex mountain terrain and forest areas. However, because the method of irregular triangulation is based on the two-dimensional neighbourhood search method, the computational complexity and algorithm complexity are relatively large. In general, due to significant elevation changes between tall buildings and vegetation and their neighbouring ground points, the algorithm filtering effect is better for abrupt mutations, but excessive error occurs when filtering low ground objects.

In summary, after the accuracy evaluation, the RMSE results show that the above method can effectively filter out the ground points and obtain higher DEM accuracy than before.

\section{ACKNOWLEDGEMENT}

This work was supported in part by the National Administration of CASM Fundamental research funds (No.7771715)

\section{REFERENCES}

LI Ying, SU Guozhong, LIN Zongjian. Study on the Method of Extracting High Precision DEM from Oblique Image. Bulletin of Surveying and Mapping, 2017( 2) : 30-34.

Peter Axelsson. DEM generation from laser scanner data using adaptive TIN models. International Archives of Photogrammetry andRemote Sensng,2000,33(B4):110-117.

FU Chunyong, YUAN Xitun, XU Ke, LI Ning. A Way to Abtain DEM Based on the High-resolution Air-borne SAR. Bulletin of Surveying and Mapping, 2012(4) : 50-51+64.

SHAO Weizhen, ZHAO Fuyan, LIANG zhaoyan. The Research of Improved Progressive Triangulated Irregular Network Densification Filtering Algorithm. Beijing Surveying and Mapping, 2016(6) : 17-21.

Sun Z C, Guo H D, Li X W. Error analysis of high precision DEM generated from airborne dual-antenna interferometric SAR data. Chinese High Technology Letters, 2012,22(2): 171-179.
Wimmer C, Siegmund R, Schwabisch M, et al. Generation of high precision DEMs of the Wadden Sea with airborne

interferometric SAR. IEEE Transactions on Geoscience and Remote Sensing ,2000,38(5):2234-2245.

Huang G M, Zhang J X, Zhao Z, et al. Research on airborne SAR interferometry mapping system. Acta Geodaetica et Cartographica Sinica,2008,37( 3) :277-279.

Sun Z C, Guo H D, Li X W,et al. DEM generation and error analysis using the first Chinese airborne dual-antenna interferometric SAR data. International Journal of Remote Sensing,2011,32( 23) : 8485-8504.

SHENG Shaohong, WAN Youchuan, GONG Hao, LA I Zulong. An Adaptive Threshold Segmentation Method Based on Spatial Statistic Theory to High-resolution Remote Sensing Change Detection. Geomatics and Information Science of Wuhan University.2009,34(8):902-905. 